\title{
COMPARISON OF NUMERICAL SIMULATIONS WITH EXPERIMENTAL MEASUREMENTS FOR THE RESPONSE OF A MODIFIED SUBMERGED HORIZONTAL CYLINDER MOORED IN WAVES
}

\author{
Ronan Costello \\ Centre for Ocean Energy Research, \\ NUI Maynooth, Ireland \\ Josh Davidson \\ Centre for Ocean Energy Research, \\ NUI Maynooth, Ireland
}

\author{
Davide Padeletti \\ Centre for Ocean Energy Research, \\ NUI Maynooth, Ireland \\ John V. Ringwood \\ Centre for Ocean Energy Research, \\ NUI Maynooth, Ireland
}

\begin{abstract}
To facilitate commercially relevant numerical design optimization in wave energy conversion accurate and validated simulations of wave body interactions are necessary. Wave energy, more so than almost any other industry, can benefit from such numerical optimization because of the high cost and long period of design iteration in experimental and field testing. For the foreseeable future wave energy device design and optimization will continue to rely heavily on potential flow solvers. Two important prerequisites to successfully using simulations based on these codes are firstly a need to validate the simulation implementation by comparison with experiment and secondly a need to supplement the potential flow solution with experimentally (or CFD) derived coefficients for the forces that are neglected by the potential flow solver. This paper attempts to address both of these prerequisites. A comparison of numerical simulations and physical wave tank experiments on a submerged horizontal cylinder moored in waves is presented. Good agreement between numerical model and experiment is achieved. At operating points where the body response is linear a numerical model based purely on potential flow and linear mooring stiffness achieves excellent results and at operating points where the body response is non-linear a time domain model with frequency independent quadratic damping is shown to give good agreement for a wide range of wave periods and amplitudes.
\end{abstract}

Keywords: Wave Energy Conversion, Numerical Modeling, Numerical Optimization, Wave Tank Experiments.

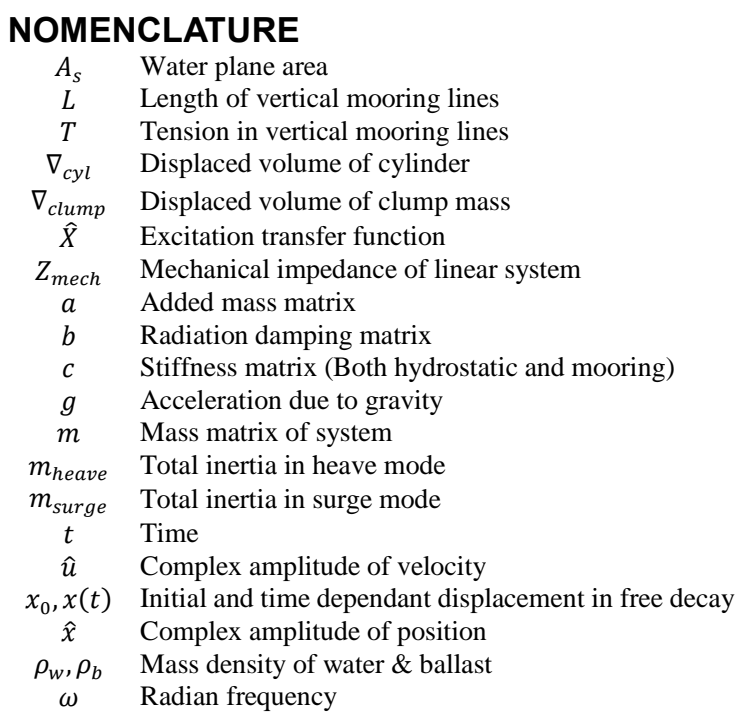

\section{INTRODUCTION}

In order to facilitate a comparison between experimental measurements and numerical simulations the response of a generic shape moored in waves was measured in a wave tank and simulated using both frequency domain and time domain methods. The shape of the wetted surface is generic; it is neither a ship nor any particular wave energy device. A horizontal cylinder is chosen because it is a generic shape that is thought to be more relevant to wave energy conversion devices than other generic shapes (such as, for example, a 
vertical cylinder). The submerged cylinder is augmented to give it a surface piercing element so that a heave resonance occurs. The moorings of the submerged cylinder are vertical lines with a clump mass arranged so that a resonance also occurs in surge. Results from two configurations are reported, the first has the heave and surge resonance frequencies well outside the frequency range of the tests, while the second has these resonances within or close to the frequency range of the test. For each of these configurations a series of free decay tests in heave and surge were undertaken followed by a series of monochromatic waves and pan chromatic sea states. The first of these configurations gives results with mild motion that are predicted very well by the results of numerical models based exclusively on potential flow theory, thereby validating our implementation. The second of these configurations gives results with more vigorous response and larger motion amplitudes, in turn leading to significant shearing and vortex sheading forces. This second set of results allows extraction of coefficients for forces not included in potential flow solutions. Use in numerical simulations of the coefficients extracted in this way gives excellent agreement between numerical and experimental results over a wide range of wave period and amplitudes.

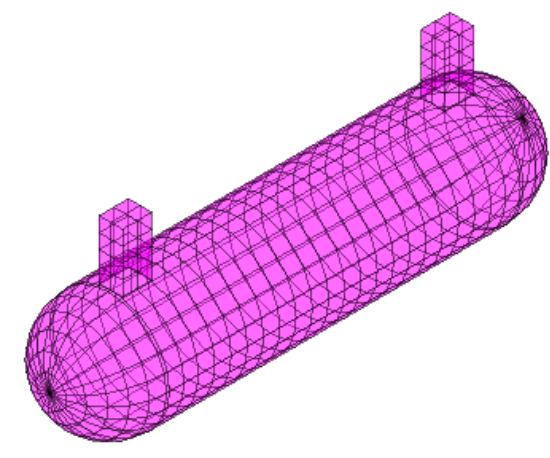

Figure 1. Submerged horizontal cylinder with domed ends and surface piercing columns.

\section{EXPERIMENTAL SETUP}

The experiments were undertaken at the Kelvin Hydrodynamics Laboratory in the University of Strathclyde, Glasgow, UK. The tank depth was $2.2 \mathrm{~m}$, tank length $76 \mathrm{~m}$, tank width $4.6 \mathrm{~m}$ with model centralized in tank. The distance from the model to the wavemaker was $37 \mathrm{~m}$.

The experimental setup comprises a submerged horizontal cylinder with domed ends, adapted to have surface piercing geometry and connected to a mooring system. Throughout the tests unidirectional waves were utilized and the model orientation was such that the wave the wave crests and cylinder axis were parallel. At rest in still water the axis of the cylinder was one diameter below the water free surface. The surface piercing geometry is provided by vertical columns of rectangular section which extend from the model upwards through the free surface. The mooring system comprises vertical lines between the cylinder and a moving clump mass and horizontal lines which restrict the motion of the clump mass to heave only. Figure 1 shows a surface model of the cylinder, Figure 2 shows a schematic of the cylinder and moorings and Figure 3 shows a photograph of the experimental setup.

The definition of modes and the sequence of modes in the matrix equations used in this paper follow the conventions shared by $[1,4]$, however, in the numerical calculations, several modes are suppressed (not calculated) so that the matrix equations in this paper are of size $3 \times 3$, these three modes are summarized in Table 1. Subscripts used in the paper, in particular with mass and stiffness matrices, refer to these modes.

Table 1. Mode Definitions

\begin{tabular}{|r|r|r|r|r|}
\hline $\begin{array}{r}\text { Mode } \\
\text { Index }\end{array}$ & $\begin{array}{r}\text { Body } \\
\text { No }\end{array}$ & Body Name & $\begin{array}{r}\text { Mode } \\
\text { Name }\end{array}$ & $\begin{array}{r}\text { Mode Index in Full } \\
\text { 6*NBODY System }\end{array}$ \\
\hline 1 & 1 & Cylinder & Surge & 1 \\
\hline 2 & 1 & Cylinder & Heave & 3 \\
\hline 3 & 2 & Clump Mass & Heave & 9 \\
\hline
\end{tabular}

\section{DEFINITION OF CONFIGURATIONS}

The stiffness and inertia of the system are such that two configurations with different behavior are possible. The surface piercing geometry gives the cylinder a stiffness in heave [1]

$$
c_{2,2}=\rho_{w} g A_{s}
$$

The cylinder and the clump mass are neutrally buoyant together but not separately, so the tension in the vertical mooring lines may be increased or decreased by moving ballast between the cylinder and the clump. The tension in the line allowing for the buoyancy of the clump mass is

$$
T=m_{3,3} g\left(1-\rho_{w} / \rho_{b}\right)
$$

$\boldsymbol{m}_{3,3}$ is the mass of the clump mass. Since both the cylinder and clump mass move together in heave the total inertia in heave is approximately independent of any such ballast transfer, however, in surge, only the cylinder and not the clump moves so the inertia in surge is a function of this mass split.

The vertical mooring lines provide a restoring force in surge; when the cylinder is moved from its equilibrium position in surge the lines are no longer vertical and a component of the tension acts to return the cylinder to its equilibrium position. The stiffness of this spring effect is

$$
c_{1,1}=T / L
$$

This is identical to the linearized surge stiffness effect experienced by a tension leg platform.

The experimental setup therefore gives an arrangement where the heave stiffness may be altered by changing the crosssectional area of the columns that form the surface piercing geometry and the surge stiffness and active inertia may be altered by moving ballast mass between the cylinder and the 
clump mass. Therefore the natural frequency in both surge and heave can be individually tailored to suit our experiments.

The flexibility in natural frequencies described in the previous paragraph is utilized to address the objectives set out in the introduction: Two configurations are defined, a first with both surge and heave resonances well outside the period range of the tests and a second with both resonances close to the period range of the tests. Table 2 gives key summary data for each configuration.

Table 2. Definition of Configurations A \& B.

\begin{tabular}{|r|r|r|l|}
\hline \multirow{2}{*}{ Quantity } & \multicolumn{2}{|c|}{ Value } & \multirow{2}{*}{ Units } \\
\cline { 2 - 3 } & Cfg. A & Cfg. B & \\
\hline Cylinder Diameter & 0.2 & 0.2 & $\mathrm{~m}$ \\
\hline Length of Cylindrical Surface & 0.6 & 0.6 & $\mathrm{~m}$ \\
\hline Length Overall & 0.8 & 0.8 & $\mathrm{~m}$ \\
\hline Submergence of Centerline & 0.2 & 0.2 & $\mathrm{~m}$ \\
\hline Column X Dimension & 0.040 & 0.112 & $\mathrm{~m}$ \\
\hline Column Y Dimension & 0.050 & 0.150 & $\mathrm{~m}$ \\
\hline Displacement of Cylinder & 23.75 & 27.00 & $\mathrm{~L}$ \\
\hline Mass of Clump Mass & 4.33 & 19.75 & $\mathrm{Kg}$ \\
\hline Length of Vert. Mooring Lines & 1.3 & 1.3 & $\mathrm{~m}$ \\
\hline Tension in Vert. Mooring Lines & 38.75 & 176.70 & $\mathrm{~N}$ \\
\hline Surge Stiffness (Moorings) & 25.8 & 135.9 & $\mathrm{~N} / \mathrm{m}$ \\
\hline Heave Stiffness (Hydrostatic) & 39.24 & 329.6 & $\mathrm{~N} / \mathrm{m}$ \\
\hline Inertia/Mass of Cylinder & 19.8 & 8.9 & $\mathrm{Kg}$ \\
\hline & & & \\
\hline
\end{tabular}

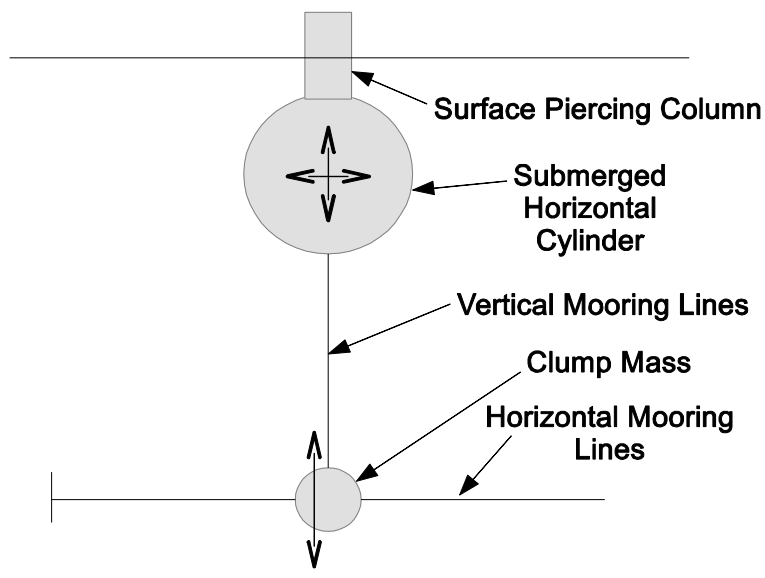

Figure 2. General Arrangement (Side View). Clump mass only moves in heave while cylinder moves in surge and heave (in pitch to a limited extent, and in sway, roll \& yaw to a very limited extent).

The ballasting of the cylinder made use of voids in the cylinder which were flooded with tank water during testing. This gave the advantage of reducing the mass of solid ballast material that was required which in turn reduced expense and allowed the model to be transported in standard airline luggage. A disadvantage of this approach is that the mass/inertia of the cylinder in the tests is not the same as its dry mass. The mass can be calculated provided the volume of the voids is known and the voids are completely filled (all air removed) when the device is deployed in the tank water.

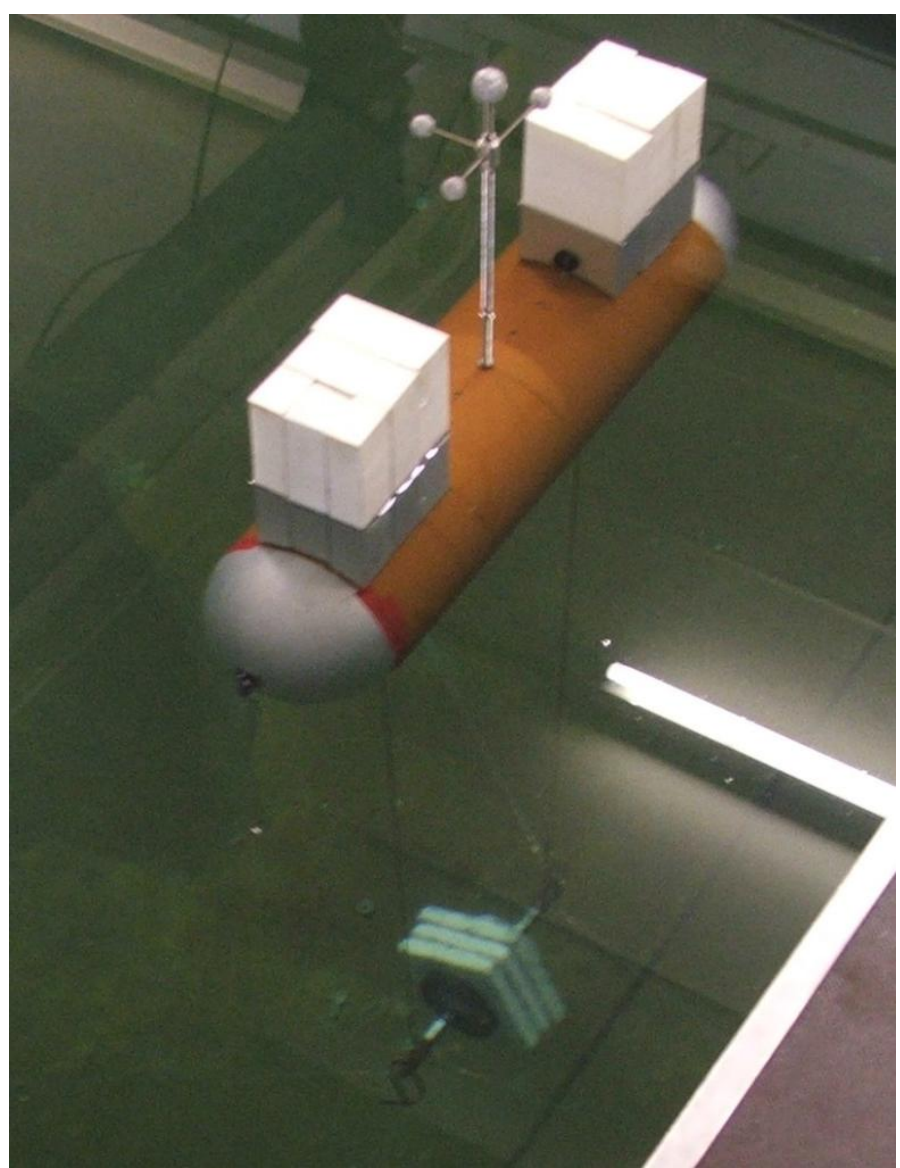

Figure 3. Photo of Model in Tank (configuration B).

\section{INSTRUMENTATION}

The instrumentation used was a six camera infrared motion tracking system which gave the 6 modes of rigid body motion of the cylinder and three wave probes which measured surface elevation. The reflectors for the camera system can be seen in position on the model in Figure 3. The wave probes were positioned, one in line with the model and one upstream of the device and a third closer to the wavemaker.

\section{DATA ANALYSIS METHODOLOGY \\ Free Decay}

For each configuration a series of free decay tests were done in heave and in surge. The objectives of these tests are to verify the inertia and stiffness and to investigate the damping characteristics in each of these modes. To this end the following tests were undertaken.

i. Heave free decay with horizontal mooring lines removed. Various initial amplitudes.

ii. Heave free decay with full moorings. Various initial amplitudes. 
iii. Surge free decay with full moorings. Various initial amplitudes.

The processing of the free decay in general followed the procedures in [2]. The following function was fitted to the decaying motion

$$
x(t)=\alpha+e^{-\beta t}\left(\gamma_{c} \cos (\omega t)+\gamma_{s} \sin (\omega t)\right)
$$

First, the stiffness and inertia in each test were verified based on an analysis of the frequency of the free decay.

In test $i$. the stiffness is purely hydrostatic and may be reliably calculated so that the total effective inertia of the system in the heave mode may then be verified by

$$
m_{\text {heave }} \approx c_{2,2} / \omega_{i}^{2}
$$

where $\omega_{i}$ is the frequency resulting from the curve fit in test $i$. In practice the displacement of the cylinder and clump mass were available by both measurement and calculation and the added mass of the cylinder is calculated by boundary element methods however the added mass of the clump mass was not calculated, so its value for the heave mode was estimated using

$$
a_{3,3}=m_{\text {heave }}-m_{2,2}-m_{3,3}-a_{2,2}
$$

Because the clump mass is deeply submerged $a_{3,3}$ is assumed to be constant, i.e. independent of frequency.

In test ii. the total effective heave stiffness is the hydrostatic stiffness plus a contribution from the horizontal mooring lines while the inertia is unchanged from test $i$. The total heave stiffness can then be estimated from

$$
c_{\text {heave }} \approx m_{\text {heave }} \omega_{\text {ii }}^{2}
$$

The heave stiffness acting on the clump mass (mode 3 ) due to the horizontal lines is then available as

$$
c_{3,3}=c_{\text {heave }}-c_{2,2}
$$

In test iii. the effective inertia in surge is calculated from

$$
m_{\text {surge }}=m_{1,1}+a_{1,1}
$$

(Note: $m_{1,1}=m_{2,2}=$ the flooded mass of the cylinder). The stiffness in the surge mode may then be deduced from the frequency of the free decay

$$
c_{1,1} \approx m_{\text {surge }} \omega_{i i i}^{2}
$$

In each of the tests in i. ii. \& iii. the linear damping necessary to cause the decay of the unforced motion was calculated from

$$
b=2 \beta m_{\text {surge }}
$$

or

$$
b=2 \beta m_{\text {heave }}
$$

Additionally the initial amplitude of the decay was calculated for each test from the parameters of the curve fit

$$
x_{0}=\sqrt{\gamma_{c}^{2}+\gamma_{s}^{2}}
$$

Plots of the resulting damping coefficients against initial amplitude are included in the results section.

\section{Monochromatic}

For each configuration a series of monochromatic tests were done with $12 \mathrm{~mm} 25 \mathrm{~mm}$ and $75 \mathrm{~mm}$ wave amplitudes. The objectives of these tests are to characterize the position response in monochromatic waves for comparison with numerical predictions and for extraction of terms to represent forces neglected by the Lapaclian flow solvers. In processing of each test the following function was fitted to the resulting motion in both surge and heave

$$
\begin{gathered}
x(t)=\alpha+\gamma_{c} \cos (\omega t)+\gamma_{s} \sin (\omega t) \\
|\hat{x}|=\sqrt{\gamma_{c}^{2}+\gamma_{s}^{2}}
\end{gathered}
$$

The amplitude of these fitted curves, $|\hat{x}|$, was normalized by dividing by the wave amplitude and the resulting ratio was plotted against period to give the so called response-amplitudeoperator (RAO) for comparison with the numerical results.

In most cases the quality of the curve fit was excellent. In a relatively small number of tests the response was irregular, with distortion or an apparent frequency doubling, in these cases no points were added to the RAO. In-depth analysis of these particular records may be the subject of further research.

\section{Panchromatic}

For each configuration a series of panchromatic tests were done with peak periods in the range 1.1 to 3.3 seconds. The objectives of these tests are to characterize the position response in panchromatic waves for comparison with numerical predictions. To facilitate this comparison the wave spectrum was characterized so that the same wave spectrum could be input to the numerical models. The wave spectrum was characterized by calculating the FFT of the wave elevation for each test and the response of the device was characterized by calculating the root-mean-square of the surge and heave motions in each sea state.

\section{NUMERICAL MODELS}

Data generated by both frequency domain and time domain simulations is used in this paper. The frequency domain results are used for comparison with the experimental measurements from configuration A where the response is expected to be linear, and the time domain results are used for comparison with experimental measurements from configuration $\mathrm{B}$ where the response is expected to be outside the linear range.

In both models the linear hydrodynamics of the cylinder are "complete" i.e. the excitation is the diffraction force represented as a frequency dependent transfer function (wave to force) and the radiation force coefficients are frequency dependent hydrodynamic added mass and radiation damping. The excitation and radiation quantities are calculated using WAMIT. The hydrodynamics of the clump mass are simplified, 
since the clump mass is deeply submerged the wave excitation is neglected, the wave radiation damping is also neglected and the hydrodynamic added mass is assumed to be independent of frequency. The hydrodynamic interactions between the cylinder and clump mass are neglected.

The boundary element solution did not exhibit any artifacts associated with so-called irregular frequencies as described in [4]. No grid independence study was undertaken so some refinement of the results might result if this were done.

\section{Frequency Domain Model}

The equation solved to arrive at the motion of the floating body at each wave frequency is [1]

$$
\hat{u}=\hat{X} / Z_{\text {mech }}
$$

Where $\hat{u}$ is the vector of complex amplitude of velocity per unit wave height, $\hat{X}$ is the excitation transfer function, and $Z_{\text {mech }}$ is the mechanical impedance matrix of the system. $Z_{\text {mech }}$ is calculated from

$$
Z_{\text {mech }}=(m+a) i \omega+b+\left(c+c_{t}\right)(i \omega)^{-1}
$$

Where $m$ is the inertia matrix, $a$ is the added mass matrix, $b$ is the radiation damping matrix, $c$ is the combined hydrostatic and mooring stiffness matrix, $c_{t}$ is the additional stiffness to represent the vertical tether between the cylinder and the clump mass, $\omega$ is the wave frequency and $i=\sqrt{-1}$. The stiffness matrix of the vertical tether, linking modes $2 \& 3$, is given by

$$
c_{t}=\left[\begin{array}{ccc}
0 & 0 & 0 \\
0 & +k & -k \\
0 & -k & +k
\end{array}\right]
$$

where for the steel ropes used $k \approx 4 \times 10^{6} \mathrm{~N} / \mathrm{m}$.

Since the comparisons later in this paper are made on the basis of position response the position amplitude must be calculated from the velocity amplitude. The complex amplitude of the body position per unit wave height is

$$
\hat{x}=\hat{u} /(i \omega)
$$

The magnitude of this complex amplitude, $|\hat{x}|$, is plotted as the frequency domain RAO in results section.

\section{Time Domain Model}

For wave periods and motion amplitudes where the behavior of the floating body is non-linear the motions of the floating body in response to the incoming waves are calculated using a modified Cummins equation [3].

$$
\begin{gathered}
\left(m+a_{\infty}\right) \ddot{\xi}+\int_{0}^{\infty} k(t-\tau) \dot{\xi}(\tau) d \tau \\
+d \dot{\xi}|\dot{\xi}|+\left(c+c_{t}\right) \xi=F_{e} \\
k(t)=\frac{2}{\pi} \int_{0}^{\infty} b(\omega) \cos (\omega t) d \omega
\end{gathered}
$$

The modification to the standard Cummins equation is the addition of a quadratic damping term, with coefficient $d$ which has units of $\mathrm{Nm}^{-2} \mathrm{~s}^{2} . a_{\infty}$ is the hydrodynamic added mass of the system as the frequency tends to infinity.

\section{Hydrodynamic Coefficients}

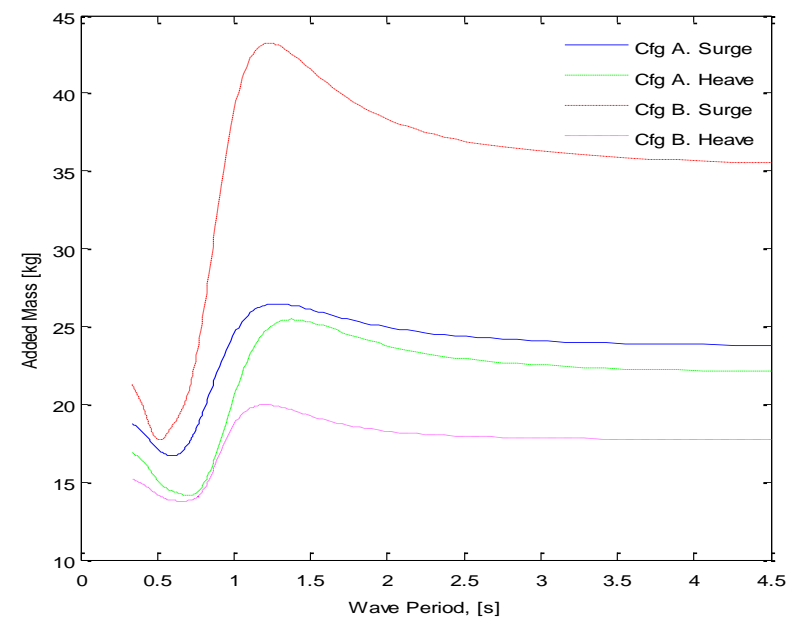

Figure 4. Added Mass. Elements from main diagonal of added mass matrix for configuration A \& $\mathrm{B}$.

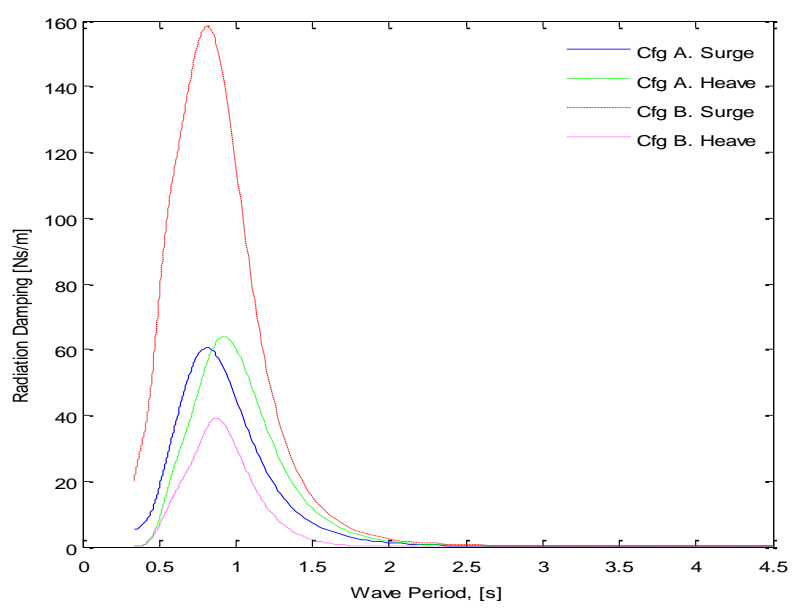

Figure 5. Radiation Damping. Elements from main diagonal of radiation damping matrix for configuration $\mathrm{A} \& \mathrm{~B}$.
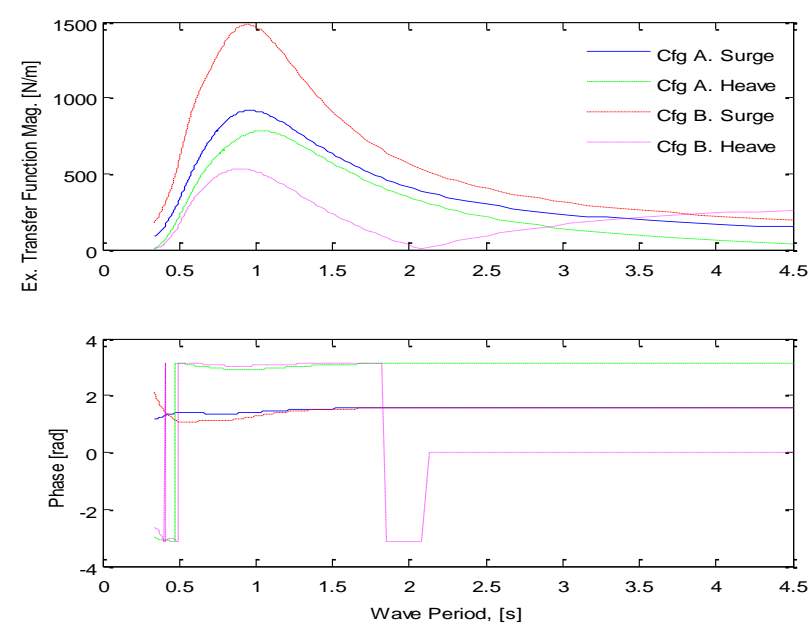

Figure 6. Excitation transfer function, magnitude (top) and phase (bottom) for surge and heave and configurations A \& B. 
The hydrodynamic coefficients for the radiation and diffraction forces were calculated using WAMIT [4]. Figure $4 \&$ Figure 5 graph the frequency dependence of the main diagonal of the added mass and radiation damping matrices respectively. Figure 6 graphs the frequency dependence of the magnitude and phase of the excitation force transfer function.

\section{FREE DECAY RESULTS (CFG A \& B)}

Table 3 summarizes the results of the investigation of inertia and stiffness from the free decay test data for both configurations. The italicized lines are of key importance to the comparison between numerical and experimental data.

Table 3. Inertia and Stiffness Verification from Free Decay.

\begin{tabular}{|c|c|c|c|c|}
\hline \multirow[t]{2}{*}{ Quantity } & \multirow{2}{*}{$\begin{array}{l}\mathrm{Eq} \\
\text { no }\end{array}$} & \multicolumn{2}{|c|}{ Value } & \multirow[t]{2}{*}{ Unit } \\
\hline & & cfg. A & cfg. B & \\
\hline \multicolumn{5}{|c|}{ Test i. Heave free decay without horizontal mooring lines } \\
\hline Number of Repeat Tests & & 2 & 2 & - \\
\hline Frequency of Free Decay & $(4)$ & 0.914 & 2.627 & $\mathrm{rad} / \mathrm{s}$ \\
\hline Period of Free Decay & & 6.87 & 2.39 & $\mathrm{~s}$ \\
\hline $\begin{array}{r}\text { Calc. Heave Hydrostatic } \\
\text { Stiffness }\end{array}$ & $(1)$ & 39.24 & 329.6 & $\mathrm{~N} / \mathrm{m}$ \\
\hline Total Inertia in Heave Mode & $(5)$ & 46.97 & 47.76 & $\mathrm{~kg}$ \\
\hline Added Mass of Clump & $(6)$ & 0.94 & 1.02 & $k g$ \\
\hline Mass of Cyl. Voids Flooded & $(9)$ & 19.80 & 8.99 & $k g$ \\
\hline \multicolumn{5}{|c|}{ Test ii. Heave free decay (with full moorings) } \\
\hline Number of Repeat Tests & & 6 & 15 & \\
\hline Frequency of Free Decay & $(4)$ & 0.947 & 2.723 & $\mathrm{rad} / \mathrm{s}$ \\
\hline Period of Free Decay & & 6.63 & 2.31 & $\mathrm{~s}$ \\
\hline Total Heave Stiffness & $(7)$ & 42.09 & 365.9 & $\mathrm{~N} / \mathrm{m}$ \\
\hline Mooring Heave Stiffness & $(8)$ & 2.85 & 36.30 & $N / m$ \\
\hline \multicolumn{5}{|c|}{ Test iii. Surge free decay (with full moorings) } \\
\hline Number of Repeat Tests & & 6 & 14 & \\
\hline Frequency of Free Decay & $(4)$ & 0.786 & 1.667 & $\mathrm{rad} / \mathrm{s}$ \\
\hline Period of Free Decay & & 7.99 & 3.77 & $\mathrm{~s}$ \\
\hline Mooring Surge Stiffness & & 26.86 & 129.1 & $N / m$ \\
\hline & & & & \\
\hline
\end{tabular}

The "Mass of Cyl. Voids Flooded" quantity agrees to within $0.005 \mathrm{~kg}$ with the estimate from our solid modeling program for both configurations. The "Added Mass of Clump" quantity was not calculated by the boundary element method (because the shape of the clump mass is somewhat irregular and in any case the value of this added mass was low) so this figure is input to the numerical model as an experimentally determined quantity. "Moorings Heave Stiffness" \& "Moorings Surge Stiffness" quantities are also added to the numerical as experimentally determined quantities. In both configurations the heave mooring stiffness is low compared to the heave hydrostatic stiffness which is as was intended in the design.
The surge mooring stiffness is about $10 \% \& 5 \%$ lower than the intended values in configuration A \& B respectively. Since the inertia of the flooded cylinder agrees so well with the anticipated value the authors are satisfied that the tension in the vertical lines is as intended. Therefore the discrepancy in the stiffness is thought to be due to a discrepancy in the length of the vertical mooring line. This could be due to an inaccuracy in the assembly of the lines or due to the attachment points not being on the cylinder center line. Since the discrepancy is not the same in both configurations it is unlikely that the latter is the sole explanation since this effect could be expected to be the same in each configuration.

The regression on the free decay data gave very consistent results in terms of frequency but less so in terms of rate of decay: The frequency of oscillation was independent of the initial displacement in the free decay while the damping coefficient was not. To investigate this further the damping coefficients from the curve fits are plotted against initial displacement in each test, Figure 7 shows these plots. Within each mode and configuration the relationship between damping and initial amplitude appears to be linear with a non-zero axis intercept. This characteristic is consistent with a combination of linear and quadratic damping forces, since purely linear damping would give zero gradient (horizontal) straight lines on this graph and purely quadratic damping would give straight lines with positive gradient and zero intercept. The heave mode in configuration $\mathrm{A}$ is close to purely linear damping while surge mode in configuration $\mathrm{B}$ is the closest to purely quadratic damping.

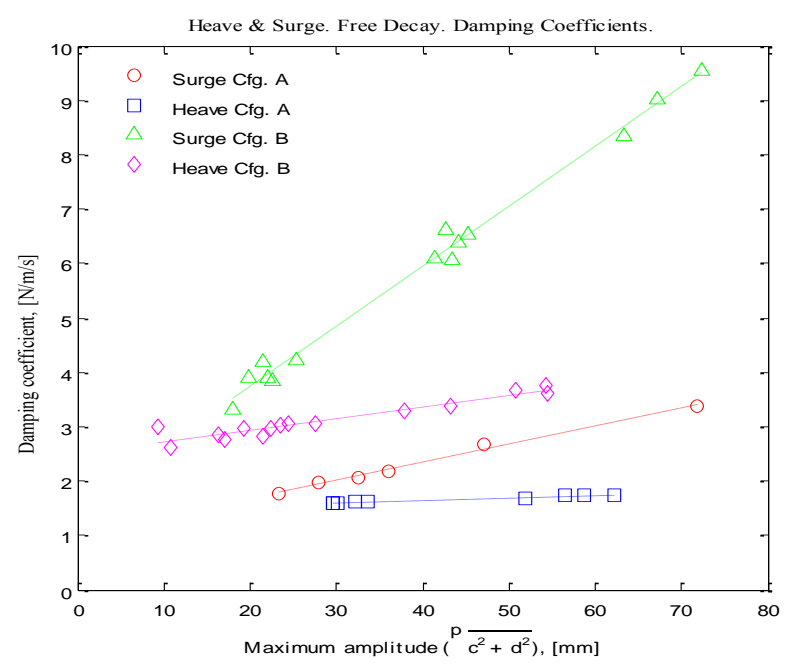

Figure 7. Damping coefficients from free decay for surge and heave modes for configurations A \& B as a function of initial displacement.

The non-zero gradient in the curve fits of Figure 7 highlight a deficiency in the structure of equation (4) which is not capable of fitting the motion which results when higher than first order damping forces are significant. 


\section{MONOCHROMATIC RESULTS (CFG A)}

Figure 8 gives a comparison of the measured and simulated ROA's for configuration A. The underlying numerical model uses only radiation diffraction solution from WAMIT and inertia and stiffness values as summarized in the free decay results section. The agreement between numerical and experimental data is excellent. At these low amplitudes viscous forces are insignificant and the assumptions underlying Laplacian flow, in particular assumptions of invicid and irrotational flow, are valid.

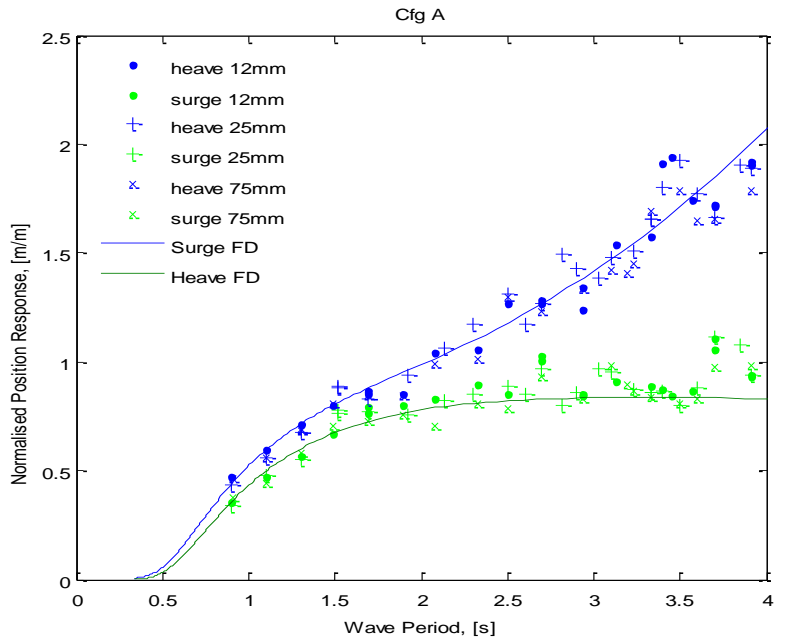

Figure 8. Comparison of measured and simulated Surge \& Heave RAO for cfg A. (FD: Frequency Domain. TD: Time domain.)

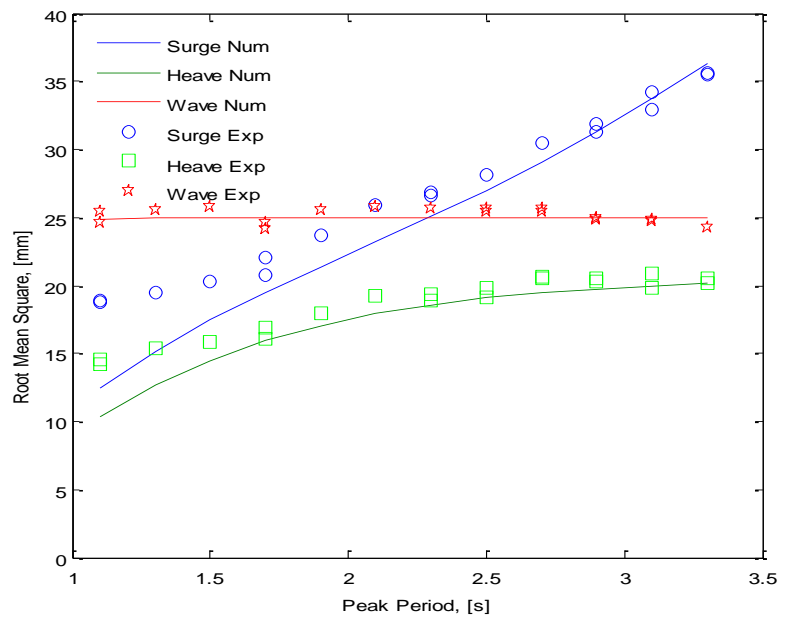

Figure 9. Comparison of standard deviation of measured and simulated signals for a range of peak periods. Configuration A. (Num: Numerical. Exp: Experimental).

\section{PANCHROMATIC RESULTS (CFG A)}

Figure 9 shows a comparison of numerical and experimental results for a series of panchromatic waves with configuration A of the model. The comparison is done on the basis of the rootmean-square of the signals. The numerical results are generated by superposition of frequency domain solutions. Good agreement is evident between measured and simulated results but there is room for further improvement. It is possible that further effort in establishing the equivalence of the inputs to the numerical and experimental systems would lead to a further improvement in this agreement.

\section{MONOCHROMATIC RESULTS (CFG B)}

Figure 10 gives a comparison of measured and simulated RAO's for surge motion of configuration B. In keeping with the design intention the magnitude of the RAO's is higher for configuration $\mathrm{B}$ than it is for configuration $\mathrm{A}$.

Three wave heights are tested and at low period the response is close to linear and the three RAO's are very close. At higher wave periods the surge motion increases so that the system no longer displays linear characteristics and the RAO values are lower for the larger waves.

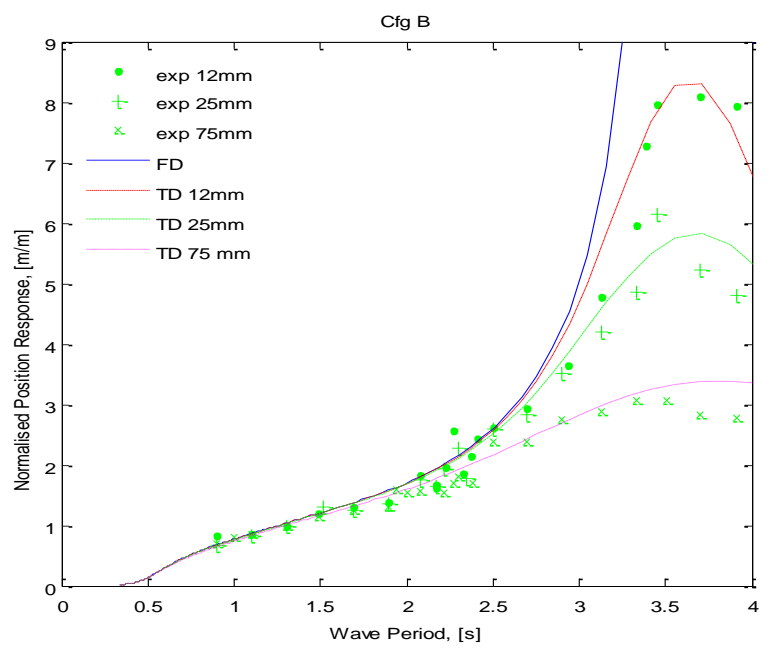

Figure 10. Comparison of measured and simulated Surge RAO for configuration B. (exp: experimental. FD: Frequency Domain. TD: Time domain. $12 \mathrm{~mm} 25 \mathrm{~mm} \& 75 \mathrm{~mm}$ indicate the wave amplitude)

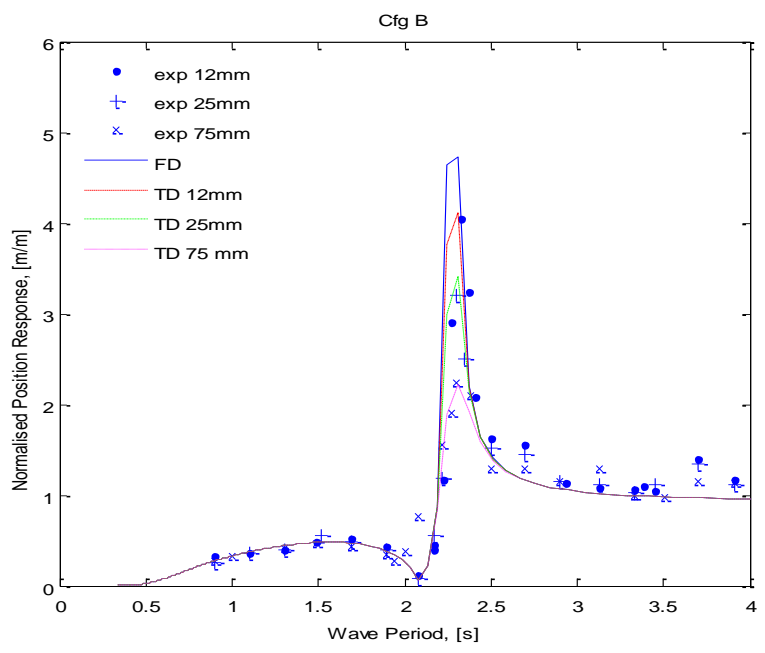

Figure 11. Comparison of measured and simulated Heave RAO for configuration B. (legend abbreviations as in Figure 10) 
The blue line in Figure 10 shows the linear frequency domain result, as might be expected it matches the experimental data quite well in the region where the system behaves linearly. However at higher periods, lower frequency, this linear result over estimates the motion significantly. The TD12 TD25 and TD75 lines are from the time domain model where a constant coefficient quadratic damping has been applied.

Figure 11 gives the results for comparison of experimental measurements of heave in configuration $\mathrm{B}$ compared to frequency and time domain results. There is a resonant peak around $2.4 \mathrm{~s}$ wave period (as predicted in Table 2). The experimental results show that the system behaves linearly before and after the resonant peak but not close to the peak. The blue line again shows the linear frequency domain results. The results from the time domain simulation match very well the experimental observations.

\section{PANCHROMATIC RESULTS (CFG B)}

Figure 12 shows a comparison of numerical and experimental results for a series of panchromatic waves for configuration $B$ of the device. The comparison again is done on the basis of the root-mean-square of the position signals. The numerical results are generated by the same time domain model used to generate the data underlying Figure 10 and Figure 11, with the same coefficients.

Similarly to Figure 9 the agreement is good but might yet be improved. As with Figure 9 this improvement might come from improving the equivalence of the input to the numerical simulation with the experimentally measured waves.

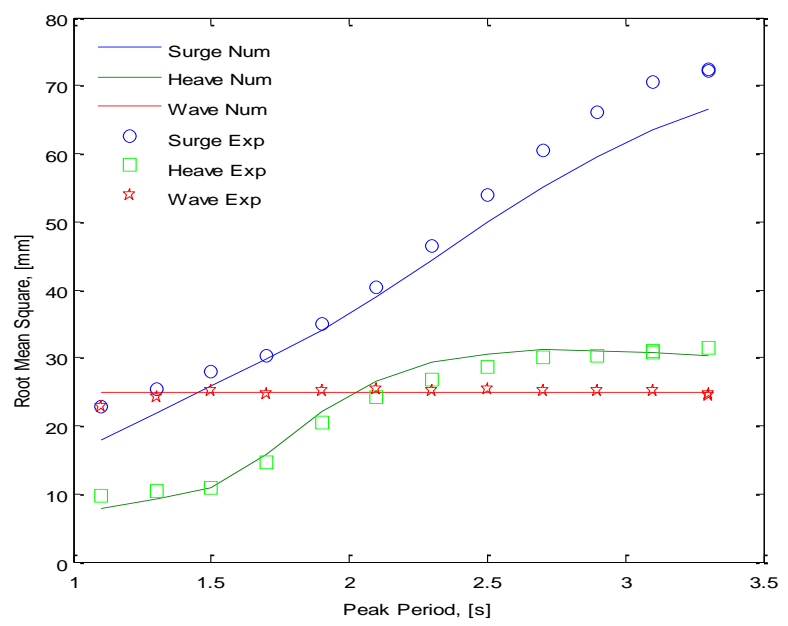

Figure 12. Comparison of standard deviation of measured and simulated signals for a range of peak periods. Configuration B. (Num: Numerical. Exp: Experimental)

\section{CONCLUSION}

The motions of a submerged horizontal cylinder moored in waves were investigated using both numerical and experimental methods and in resonant and non-resonant configurations.
The results show that the simulations based on linear potential theory agree very well with the experimental observations at operating points that are away from the resonant response of the body. With this observation the implementation of these simulations is therefore validated.

The linear model over predicts the motions of the bodies at wave periods close to the natural period. In this case the addition of a quadratic damping term to the time domain simulation gives very good agreement with experiment. Within the wave period and amplitude range tested the comparison indicates that a quadratic damping coefficient that is independent of both wave period and amplitude is appropriate.

Caution is, however, necessary in utilizing numerical models with experimentally derived coefficients in design optimization as the dependence of the experimentally derived coefficients on the design variables to be optimized must be understood.

No power take off forces were present in this experiment. Inclusion of PTO forces will alter the relative importance of the quadratic damping forces so that even though a real WEC might operate close to resonance in one or more modes the importance of the quadratic damping in a real WEC could be expected to be intermediate to that in the two configurations presented in this paper.

The demonstrated level of agreement between simulation and experiment is a prerequisite for numerical optimization of wave energy converters. The work reported in this paper is a step towards numerical optimization of wave energy converters based on validated numerical simulations.

\section{ACKNOWLEDGMENTS}

The financial support of Enterprise Ireland grant number CF/2012/2017 is gratefully acknowledged.

MARINET FP7 trans-national infrastructure access support (project acronym "COERverFB"), is gratefully acknowledged.

\section{OPEN DATA}

The data recorded in this work is to be made available under an open data arrangement. Please contact the author for more information.

\section{REFERENCES}

[1] J. Falnes. "Ocean Waves and Oscillating Systems". (Cambridge: Cambridge University press, 2002).

[2] J.M.J Journee, W.W. Massie "Offshore Hydromechanics Manual". (Delft Technical University, 2001).

[3] T.I. Fossen "Handbook of Marine Craft Hydrodynamics and Motion Control” (Wiley-Blackwell, 2011).

[4] "WAMITv7 Manual”, WAMIT Inc. 